\title{
AGREGAÇÃO E FRAÇÕES FÍSICAS DA MATÉRIA ORGÂNICA DE UM ARGISSOLO VERMELHO SOB SISTEMAS DE USO NO BIOMA PAMPA ${ }^{(1)}$
}

\author{
Daiane Carvalho dos Santos ${ }^{(2)}$, Clenio Nailto Pillon ${ }^{(7)}$, Carlos Alberto Flores ${ }^{(7)}$, \\ Cláudia Liane Rodrigues de Lima ${ }^{(3)}$, Estela Mari Cunha Cardoso ${ }^{(4)}$, Betânia \\ Fraga Pereira $^{(6)}$ \& Antonio Salvio Mangrich ${ }^{(5)}$
}

\begin{abstract}
RESUMO
O emprego de sistemas agrossilvipastoris tem sido importante estratégia de uso de solos, principalmente arenosos pertencentes ao bioma Pampa. Objetivouse, neste estudo, avaliar a agregação, o teor de C orgânico total (COT) e de N total (NT), o teor de C nas frações físicas e o grau de humificação da MO em um Argissolo Vermelho eutrófico arênico submetido a diferentes sistemas de uso no município de Alegrete, RS. As avaliações foram desenvolvidas em uma floresta homogênea de eucalipto (FH), na entrelinha do sistema agrossilvipastoril sob pastagem (SA) e em campo nativo $(\mathrm{CN})$, nas camadas de $0,000-0,025 \mathrm{~m} ; 0,025-0,075 \mathrm{~m} ; 0,075-0,125 \mathrm{~m}$; e de 0,125-0,225 m. Foram coletadas amostras deformadas de solo para a avaliação de agregados estáveis em água (AEA, \%) e dos teores de COT e de NT. Foi realizado o fracionamento físico granulométrico e densimétrico da MO, sendo as frações leve livre (FLL) e fração leve oclusa (FLO) da camada superficial submetidas às análises de Infravermelho com Transformada de Fourier (FTIR) e Ressonância Paramagnética Eletrônica (EPR). Os AEA em todas as camadas e o diâmetro médio ponderado na camada de $0,025-0,075 \mathrm{~m}$ indicaram maior degradação estrutural do solo na entrelinha do SA. Teores mais elevados de COT, NT, C da fração grosseira
\end{abstract}

(1) Parte da Tese Doutorado do primeiro autor apresentada ao Programa de Pós-Graduação em Agronomia da Universidade Federal de Pelotas - UFPel. Recebido para publicação em 20 de maio de 2010 e aprovado em 27 de julho de 2011.

(2) Pós-Doutoranda da Universidade Federal de Pelotas - UFRGS, Av. Bento Gonçalves 7712, CEP 90540-000 Porto Alegre (RS).E-mail: santos.daianec@gmail.com

(3) Professora do Departamento de Solos, Universidade Federal de Pelotas - UFPel. Caixa Postal 354, CEP $96010-900$ Pelotas (RS). E-mail: clrlima@yahoo.com.br

(4) Graduanda em Química (Departamento de Química/UFPR) no Centro Politécnico. Jardim das Américas, CEP 81531-990 Curitiba (PR). E-mail: estelamcc@hotmail.com

(5) Professor Sênior Departamento de Química, Universidade Federal do Paraná - UFPR. Rua dos Funcionários 1540, CEP 80035050 Curitiba (PR). Email: mangrich@quimica.ufpr.br

(6) Pesquisadora FAPEG/Embrapa Clima Temperado. E-mail: betaniapereira@yahoo.com.br

(7) Pesquisadores da Embrapa Clima Temperado. Rod. BR 392, km 78, Caixa Postal 403, CEP 96001-970 Pelotas (RS). E-mails: clenio.pillon@cpact.embrapa.br; flores@cpact.embrapa.br 
(CFG), C associado aos minerais (CAM), FLL e FLO na camada superficial foram observados na FH. Entre os usos do solo, os espectros de FTIR foram semelhantes, contudo a FLL apresentou bandas mais intensas na região de $1.072 \mathrm{~cm}^{-1}$, sugerindo maiores quantidades de polissacarídeos em relação à FLO. As densidades de spins obtidas por EPR na FLO foram maiores em relação à FLL, indicando maior humificação desta fração.

Termos de indexação: eucalipto, fracionamento físico, solos arenosos.

\title{
SUMMARY: AGGREGATION AND PHYSICAL FRACTIONS OF ORGANIC MATTER IN AN ALFISOL UNDER DIFFERENT USE SYSTEMS IN THE PAMPA BIOME
}

\begin{abstract}
Pasture-agroforestry systems have become an important strategy for the use of sandy soils in the Pampa Biome. The purpose of this study was to evaluate the aggregation, total organic $C$ (TOC) and total nitrogen (TN), carbon of the physical fractions and humification degree of organic matter (OM). Evaluations were conducted in an Alfisol under homogenous eucalyptus forest $(\mathrm{HF})$, between the rows of a pasture-agroforestry system (PA) and in native pasture $(N P)$ in the layers 0.000-0.025, 0.025-0.075, 0.075-0.125, and 0.125-0.225 m. Disturbed samples were collected to evaluate water-stable aggregates (WSA \%), TOC and TN. The physical (granulometrical and densimetrical) fractionation of OM was carried out, and the free light fraction $(F L F)$ and occluded light fraction $(O L F)$ of OM in the surface layer subjected to Fourier Transform Infrared (FTIR) and Electron Paramagnetic Resonance (EPR) analyses. The WSA and mean weight diameter in the layer 0.025-0.075 $\mathrm{m}$ indicated a greater degradation of the PA soil. Higher levels of TOC, TN, coarse fraction carbon (CFC), carbon associated with minerals (CAM), FLF and OLF in the surface layer were observed in HF. Between land uses, the FTIR spectra were similar. However, the FLF had more intense bands in the region of $1072 \mathrm{~cm}^{-1}$, suggesting higher amounts of polysaccharides than in the $O L F$. The spin densities obtained by EPR at OLF were higher than of FLF, indicating a higher humus fraction.
\end{abstract}

Index terms: eucalyptus, physical fractionation, sandy soils.

\section{INTRODUÇÃO}

A região Sudoeste do Rio Grande do Sul apresenta alta vulnerabilidade socioeconômica, por possuir extensas áreas de formações areníticas, as quais, devido à redução da cobertura vegetal, sofrem alterações na vegetação. $\mathrm{O}$ consequente aumento da erosão resulta na degradação desses solos e do ecossistema regional. A fragilidade natural dos solos, aliada à baixa aptidão agrícola e ao uso tradicional para a criação extensiva de gado, tem acelerado o processo de erosão, ampliando as áreas com vegetação rarefeita e campos arenizados (Ribaski et al., 2005).

Os sistemas agrossilvipastoris têm sido preconizados como alternativa de uso sustentável, principalmente em áreas sujeitas à degradação do solo. A deposição de folhas da parte aérea dos cultivos pode influenciar na quantidade e disponibilidade de nutrientes pelo aporte de matéria orgânica (MO) (Ribaski et al., 2009). A MO constitui um dos atributos de qualidade do solo, porque indica as alterações nas condições ambientais e no manejo. Alterações promovidas na distribuição e estabilidade de agregados e no teor de C orgânico total (COT) e de N total (NT) constituem indicadores sensíveis às práticas de manejo no médio e no longo prazo. Em curto prazo, alterações nas proporções das frações lábeis da $\mathrm{MO}$, como o C da fração grosseira (CFG) e, ou, fração leve livre, (FLL) e leve oclusa (FLO) podem fornecer informações importantes sobre a qualidade do solo, permitindo correções nas estratégias de uso e de manejo adotadas.

Associado ao fracionamento físico, têm sido utilizadas diferentes técnicas espectroscópicas que permitem informações importantes sobre a qualidade e humificação da MO. A espectroscopia de Infravermelho com Transformada de Fourier por Transmitância (FTIR) é uma técnica rápida, econômica e muito utilizada em Ciência do Solo (Russell \& Fraser, 1994). A espectroscopia de FTIR baseia-se no fato de que os diversos tipos de ligações químicas e de estruturas moleculares numa molécula absorvem radiação eletromagnética na região do infravermelho, em comprimentos de onda característicos, e, como consequência, os átomos envolvidos entram em vibração. Em espectrofotômetro de FTIR, a percentagem de radiação transmitida pela amostra num intervalo de comprimento de onda de 
2,5 a $25 \mu \mathrm{m}$ (infravermelho fundamental, número de

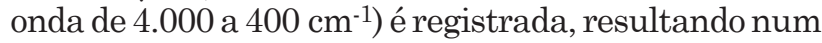
espectro contínuo de bandas de absorção (Ceretta et al., 2008). Na análise de amostras de solo e, ou, MO, essa espectroscopia é utilizada para identificar grupos carboxilas, hidroxilas, aminas, amidas, estruturas alifáticas e aromáticas, entre outros (Stevenson, 1994). A técnica de Ressonância Paramagnética Eletrônica (EPR) é utilizada na quantificação do número de radicais livres orgânicos (RLO) do tipo semiquinona (Martin-Neto et al., 1991). Ela tem sido utilizada em estudos de MO, em amostras de solo fisicamente fracionadas (Martin-Neto et al., 1994; Bayer, 1996), com o objetivo principal de diminuir o risco de alterações da $\mathrm{MO}$, as quais podem ocorrer no fracionamento químico.

Quanto maior a concentração RLO do tipo semiquinona, obtido pela técnica de EPR, maior a humificação da MO (Martin-Neto et al., 1991). Normalmente, o caráter mais humificado está relacionado com a formação de compostos mais condensados (Martin-Neto et al., 1998). Analisando um Cambissolo na camada superficial, Bayer et al. (2003) observaram menor concentração de RLO do tipo semiquinona de um solo sob plantio direto $\left(15,83 \times 10^{17}\right)$, quando comparado ao preparo convencional (18,33 x $\left.10^{17}\right)$, o que indica maior grau de humificação da MO nesse sistema em decorrência do preparo do solo.

Considerando limitado o número de trabalhos nesta área, o objetivo deste estudo foi avaliar a agregação, teor de $\mathrm{C}$ orgânico total, de $\mathrm{N}$ total e de $\mathrm{C}$ nas frações físicas, bem como a contribuição relativa dos mecanismos de proteção e o grau de humificação da MO em um Argissolo Vermelho submetido a sistemas de uso.

\section{MATERIAL E MÉTODOS}

O estudo foi realizado na estância Sá Brito, no município de Alegrete, RS, cujo solo foi classificado como Argissolo Vermelho eutrófico arênico A moderado, textura arenosa/média fase relevo suave ondulado (Embrapa, 2006). A implantação dos sistemas ocorreu em julho de 2002 em campo nativo, sendo a coleta efetuada em fevereiro de 2007.

A área sob floresta homogênea de eucalipto $(\mathrm{FH})$ estava localizada nas coordenadas $29^{\circ} 59^{\prime} 55^{\prime \prime} \mathrm{S}$ e $55^{\circ} 47^{\prime} 29^{\prime}$ "W e representava um florestamento homogêneo de Eucalyptus grandis, implantado em uma área de aproximadamente 1 ha. $\mathrm{O}$ espaçamento de plantio foi de $3 \mathrm{~m}$ e entrelinhas de $1,5 \mathrm{~m}$ entre plantas, sendo a densidade do eucalipto de 1.111 plantas ha-1. A amostragem de solo nessa área foi realizada na entrelinha de plantio do eucalipto.

A área sob sistema agrossilvipastoril (SA) estava localizada nas coordenadas $29^{\circ} 59^{\prime} 53^{\prime \prime}$ S e $55^{\circ} 47^{\prime} 34^{\prime \prime}$ W. Nesse sistema foram utilizadas linhas de plantio triplas de Eucalyptus grandis, distanciadas de 3 em $3 \mathrm{~m}$; o espaçamento entre plantas nas linhas foi de 1,5 m. As linhas triplas de plantio de Eucalyptus grandis foram distanciadas entre si por uma faixa de $14 \mathrm{~m}$ (entrelinha do sistema agrossilvipastoril). Nessa faixa de $14 \mathrm{~m}$, nos dois primeiros anos da implantação dos sistemas de uso o produtor optou pelo cultivo de grãos em rotação de aveia (Avena L.), sorgo (Sorghum bicolor L.) e milho (Zea mays) em plantio direto. Nos anos seguintes, foi cultivada a grama-forquilha (Paspalum notatum Flugge) e aveia-preta (Avena stringosa) como coberturas de inverno, milheto (Pennisetum glaucum) e consórcio de pensacola (Paspalum notatum Saurae) + braquiária brizanta (Brachiaria brizantha) como coberturas de verão. A densidade do eucalipto neste sistema é de 1.000 plantas ha-1, distribuídas em uma área de aproximadamente 4 ha. As amostras de solo nessa área foram coletadas na entrelinha, ou seja, nos $14 \mathrm{~m}$, cujas espécies de forrageiras eram utilizadas na pecuária extensiva. A intensidade de pastejo era de uma cabeça de gado por hectare.

A área de campo nativo $(\mathrm{CN})$ era adjacente às demais, estava localizada nas coordenadas $29^{\circ} 59$ '57" $\mathrm{S}$ e $55^{\circ} 47^{\prime} 29^{\prime \prime}$ W e representava cobertura remanescente de vegetação nativa da região, composta principalmente por grama-forquilha (Paspalum notatum Flugge). Assim como o sistema agrossilvipastoril, essa área apresentava-se em uso pela pecuária, sendo a intensidade de pastejo de uma cabeça de gado por hectare.

Em três trincheiras alocadas aleatoriamente foram coletadas amostras de solo em quatro camadas $(0,000$ 0,$025 ; 0,025-0,075 ; 0,075-0,125 ;$ e $0,125-0,225$ m). As amostras deformadas foram utilizadas nas análises de granulometria, agregados estáveis em água (AEA, \%), quantificação dos teores de COT, NT e fracionamento físico da MO, enquanto as indeformadas, para avaliação da densidade. A granulometria (Quadro 1), os AEA e a densidade do solo foram realizadas conforme Embrapa (1997).

Para as determinações de COT, NT e fracionamento da MO, as amostras foram secas à sombra e destorroadas manualmente de forma suave, para não provocar compactação ou ruptura dos agregados. Posteriormente, as amostras de solo foram peneiradas em malha de $8,00 \mathrm{~mm}$ e divididas em duas partes, sendo a primeira moída em almofariz de ágata para a determinação de COT e de NT e a outra, destinada ao fracionamento físico granulométrico (Cambardella \& Elliott, 1992) e densimétrico. O C oriundo do material retido na peneira $(0,053 \mathrm{~mm})$ correspondeu ao CFG, enquanto o C associado aos minerais (CAM) foi obtido pela diferença entre o COT e o CFG. Devido ao fato de maior aporte de resíduos culturais ocorrer nas camadas superficiais, o fracionamento físico densimétrico da $\mathrm{MO}$ foi realizado nas camadas entre 0,000 e 0,075 m, obtendo-se a FLL e FLO conforme Golchin et al. (1994) e Conceição et al. (2008), utilizando politungstato de sódio (PTS) com densidade de 
Quadro 1. Teores de areia, silte e argila $\left(\mathrm{g} \mathrm{kg}^{-1}\right)$ e classe textural de um Argissolo Vermelho sob sistemas de uso e camadas. Média de três repetições

\begin{tabular}{ccccc}
\hline Uso do solo $^{(1)}$ & Areia & Silte & Argila & $\begin{array}{c}\text { Classe } \\
\text { textural }\end{array}$ \\
\hline \multicolumn{5}{c}{$0,000-0,025 \mathrm{~m}$} \\
FH & 883,67 & 41,90 & 76,43 & Areia \\
SA & 892,67 & 43,43 & 63,90 & Areia \\
CN & 886,33 & 57,90 & 55,77 & Areia \\
\multicolumn{5}{c}{$0,025-0,075 \mathrm{~m}$} \\
FH & 898,67 & 25,83 & 77,50 & Areia \\
SA & 908,33 & 27,23 & 64,43 & Areia \\
CN & 897,67 & 30,17 & 72,17 & Areia \\
\multicolumn{5}{c}{$0,075-0,125 \mathrm{~m}$} \\
FH & 897,75 & 22,32 & 79,93 & Areia \\
SA & 917,50 & 13,03 & 69,47 & Areia \\
CN & 902,50 & 25,07 & 72,43 & Areia \\
\multicolumn{5}{c}{$0,125-0,225 \mathrm{~m}$} \\
FH & 912,75 & 15,95 & 71,30 & Areia \\
SA & 911,17 & 25,13 & 63,70 & Areia \\
CN & 894,08 & 24,85 & 81,07 & Areia \\
\hline
\end{tabular}

(1) FH: Floresta homogênea de eucalipto; SA: Sistema agrossilvipastoril na entrelinha; e $\mathrm{CN}$ : campo nativo.

2,0 $\mathrm{Mg} \mathrm{m}^{-3}$. Dez gramas de solo foram adicionados a $80 \mathrm{~mL}$ de solução de PTS em tubo de centrífuga de $100 \mathrm{~mL}$. O tubo foi fechado com rolha e invertido lenta e manualmente por cinco vezes, visando à liberação da FLL e evitando o rompimento dos agregados. A suspensão foi centrifugada a $2.000 \mathrm{~g}$ por $60 \mathrm{~min}$, e o sobrenadante com a FLL foi filtrado, sob vácuo, em filtro Whatman GF/C previamente quantificado quanto à sua massa. Para retirar o excesso de PTS, o filtro + FLL foi lavado com água destilada, seco a $60^{\circ} \mathrm{C}$ por 24 h e quantificada a massa de filtro + FLL. Para separar a FLO, a solução de PTS foi retornada ao tubo que continha o pellet, o qual foi suspenso novamente e submetido à dispersão com ultrassom, mediante a energia de $250 \mathrm{~J} \mathrm{~mL}^{-1}$, as quais foram determinadas previamente de forma a garantir a dispersão total dos agregados do solo em partículas primárias. Após a dispersão, a suspensão foi novamente centrifugada (2.000 g por $60 \mathrm{~min}$ ) e a FLO, obtida por filtragem, da mesma forma que a FLL. O C da fração pesada (FP) foi obtido por diferença entre o COT do solo e o C da FLL adicionado do $\mathrm{C}$ da FLO. Os teores de COT e de NT presentes no solo, no CFG, na FLL e na FLO foram quantificados por oxidação a seco, em analisador elementar LECO, sendo os resultados expressos em teor de $\mathrm{C} \mathrm{e} \mathrm{N}\left(\mathrm{g} \mathrm{dm}^{-3}\right)$, considerando-se a densidade de cada camada do solo.

As amostras do fracionamento físico densimétrico (FLL e FLO) da camada de 0,000-0,025 m foram submetidas ao tratamento com solução aquosa de HF $10 \%$ (Dick et al., 2005). Em seguida, foram realizadas as análises de Infravermelho com Transformada de
Fourier (FTIR) e de Ressonância Paramagnética Eletrônica (EPR). Os espectros de FTIR por absorbância foram registrados em espectrofotômetro Bomem FTIR, série MB100, utilizando-se pastilhas preparadas com misturas de $1 \mathrm{mg}$ de amostra e $99 \mathrm{mg}$ de $\mathrm{KBr}$ de grau espectroscópico. Para cada espectro foram somadas 32 varreduras com resolução de $2 \mathrm{~cm}^{-1}$ na região de 4.000 a $400 \mathrm{~cm}^{-1}$. Para a análise de EPR, amostras das frações FLL e FLO foram finamente moídas e colocadas em tubos de quartzo de $4 \mathrm{~mm}$ de diâmetro. Os espectros foram registrados em temperatura ambiente ( $300 \mathrm{~K})$ e de $\mathrm{N}_{2}$ líquido $(77 \mathrm{~K})$, utilizando-se espectrômetro BRUKER EMX micro operando em banda-X $(9,5 \mathrm{GHz})$, mediante o emprego de $100 \mathrm{kHz}$ de frequência de modulação. Os dados de EPR foram submetidos à simulação e ao tratamento com o auxílio dos programas Win-EPR ${ }^{\circledR}$ e SimFonia ${ }^{\circledR}$.

Os resultados foram submetidos à análise de variância e comparados pelo teste t e pela diferença mínima significativa a $5 \%$.

\section{RESULTADOS E DISCUSSÃO}

De modo geral, em todas as camadas observou-se redução da porcentagem de agregados estáveis em água (AEA), nas classes de 2,00 a 0,50 mm (Figura 1), sendo as maiores percentagens verificadas na classe $<0,25 \mathrm{~mm}$. A maior porcentagem de AEA nessa classe advém, possivelmente, do elevado teor de areia, o que afeta a formação de agregados estáveis de maior tamanho.

Entre os sistemas de uso e camadas, o sistema agrossilvipastoril (SA) foi o que apresentou maior proporção de agregados na classe de diâmetro $<0,25 \mathrm{~mm}$ (Figura 1), possivelmente relacionado ao cultivo a que essas áreas foram submetidas nos dois primeiros anos. Segundo Rozane et al. (2010), a maior percentagem de agregados nas classes de menor diâmetro pode ser justificada por práticas utilizadas durante o cultivo, o que pode levar à destruição dos agregados e à oxidação da MO. De forma geral, nas classes de agregados entre 8,00 e 2,00 mm as maiores percentagens de AEA foram verificadas no $\mathrm{CN}$ e na FH. Salton et al. (2008) relataram que sistemas de uso que proporcionem agregados mais resistentes são desejáveis, pois mantêm a estrutura do solo quando submetida à determinada força externa. $\mathrm{O}$ valor de DMP foi influenciado pelo uso somente na camada de 0,025-0,075 m (Quadro 2), decrescendo na ordem $\mathrm{FH}>\mathrm{CN}>\mathrm{SA}$. O menor DMP observado no sistema SA pode estar relacionado à compactação pelo pastejo e, ou, ao cultivo da área nos dois primeiros anos, corroborando informações de Salton et al. (2008).

Os diferentes sistemas de uso na camada de 0,000 0,025 m influenciaram o COT, NT, CFG, CAM, FLL e FLO (Quadro 3), sendo os teores mais elevados na FH justificados possivelmente pelo maior aporte de 


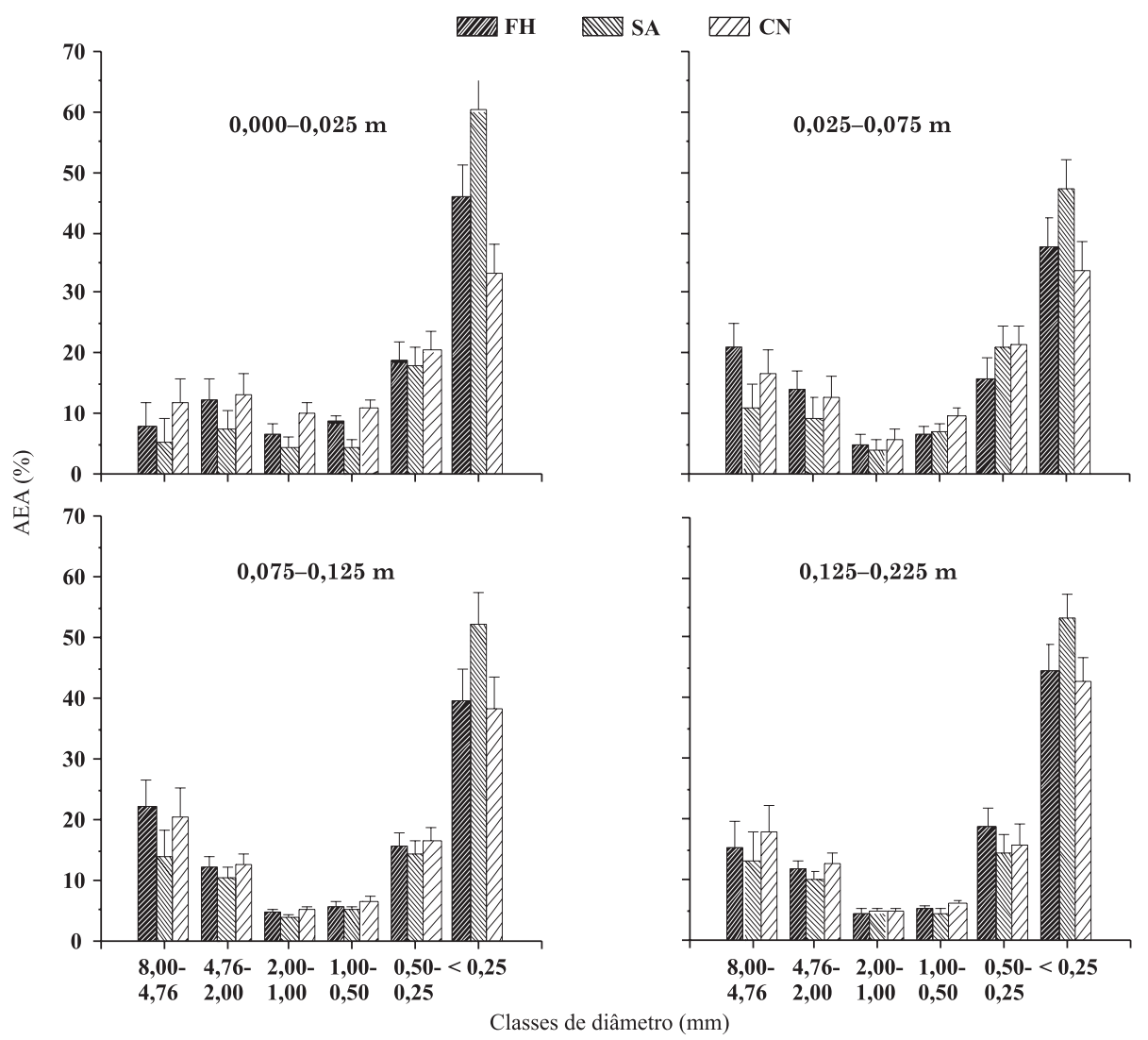

Figura 1. Agregados estáveis em água (AEA) em diferentes classes de um Argissolo Vermelho submetido a diferentes sistemas de uso e camadas. Barras verticais indicam a diferença mínima significativa a $5 \%$ entre sistemas de uso em cada classe de agregados.

Quadro 2. Diâmetro médio ponderado de agregados estáveis (DMP) de um Argissolo Vermelho submetido a diferentes sistemas de uso e camadas

\begin{tabular}{ccccc}
\hline \multirow{2}{*}{ Sistema de uso $^{(1)}$} & \multicolumn{4}{c}{ Camada } \\
\cline { 2 - 5 } & $\mathbf{0 , 0 0 0 - 0 , 0 2 5} \mathbf{~ m}$ & $\mathbf{0 , 0 2 5 - 0 , 0 7 5 ~ \mathbf { ~ }}$ & $\mathbf{0 , 0 7 5 - 0 , 1 2 5} \mathbf{~ m}$ & $\mathbf{0 , 1 2 5 - 0 , 2 2 5} \mathbf{~ m}$ \\
\hline FH & $1,22 \mathrm{a}$ & $2,04 \mathrm{a}$ & $2,04 \mathrm{a}$ & $1,60 \mathrm{a}$ \\
SA & $0,84 \mathrm{a}$ & $1,28 \mathrm{~b}$ & $1,45 \mathrm{a}$ & $1,40 \mathrm{a}$ \\
CN & $1,56 \mathrm{a}$ & $1,78 \mathrm{ab}$ & $1,98 \mathrm{a}$ & $1,80 \mathrm{a}$ \\
\hline
\end{tabular}

(1) FH: Floresta homogênea de eucalipto; SA: Entrelinha do sistema agrossilvipastoril; CN: Campo nativo. Médias seguidas pela mesma letra minúscula na coluna em cada camada não diferem entre si pelo teste $t$, que considera diferença mínima significativa a $5 \%$.

resíduos culturais depositados na superfície, comparativamente às demais.

Pulrolnik et al. (2009) verificaram aumento nos teores de COT e da FLL em um Latossolo sob eucalipto, cujo aporte de resíduos culturais e de matéria seca da serapilheira foi de 46 e $64 \%$ superior ao Cerrado e à pastagem, respectivamente. Comportamento semelhante foi observado por Lima et al. (2008) em Latossolo, em que os maiores teores de COT foram obtidos sob o eucalipto em relação à pastagem. Porém, de forma oposta a este trabalho, não foram observadas diferenças nos teores da FLO entre os diferentes sistemas de uso.
$\mathrm{Na}$ camada superficial, menores teores de COT, NT, CAM e FLO foram verificados na entrelinha do $\mathrm{SA}$ em relação à $\mathrm{FH}$ (Quadro 3), justificados possivelmente pelas reduções no aporte de resíduos da biomassa vegetal e, ou, consequência do cultivo nos dois primeiros anos. Neves et al. (2004) também encontraram menores teores de COT em SA, atribuindo o efeito ao pastejo, e redução da biomassa vegetal.

Na camada de 0,025 a 0,075 m, também foram observados menores teores de COT, NT e FLL no SA, enquanto o CFG foi maior na FH e a FLO no CN e na FH, respectivamente (Quadro 3). Guo \& Gifford (2002) 
Quadro 3. Teores de carbono orgânico total (COT), nitrogênio total (NT), relação carbono/nitrogênio (C/N), carbono na fração grosseira (CFG), carbono associado aos minerais (CAM), carbono na fração leve livre (FLL), carbono na fração leve oclusa (FLO) e carbono na fração pesada (FP) de um Argissolo Vermelho submetido a diferentes sistemas de uso e camadas

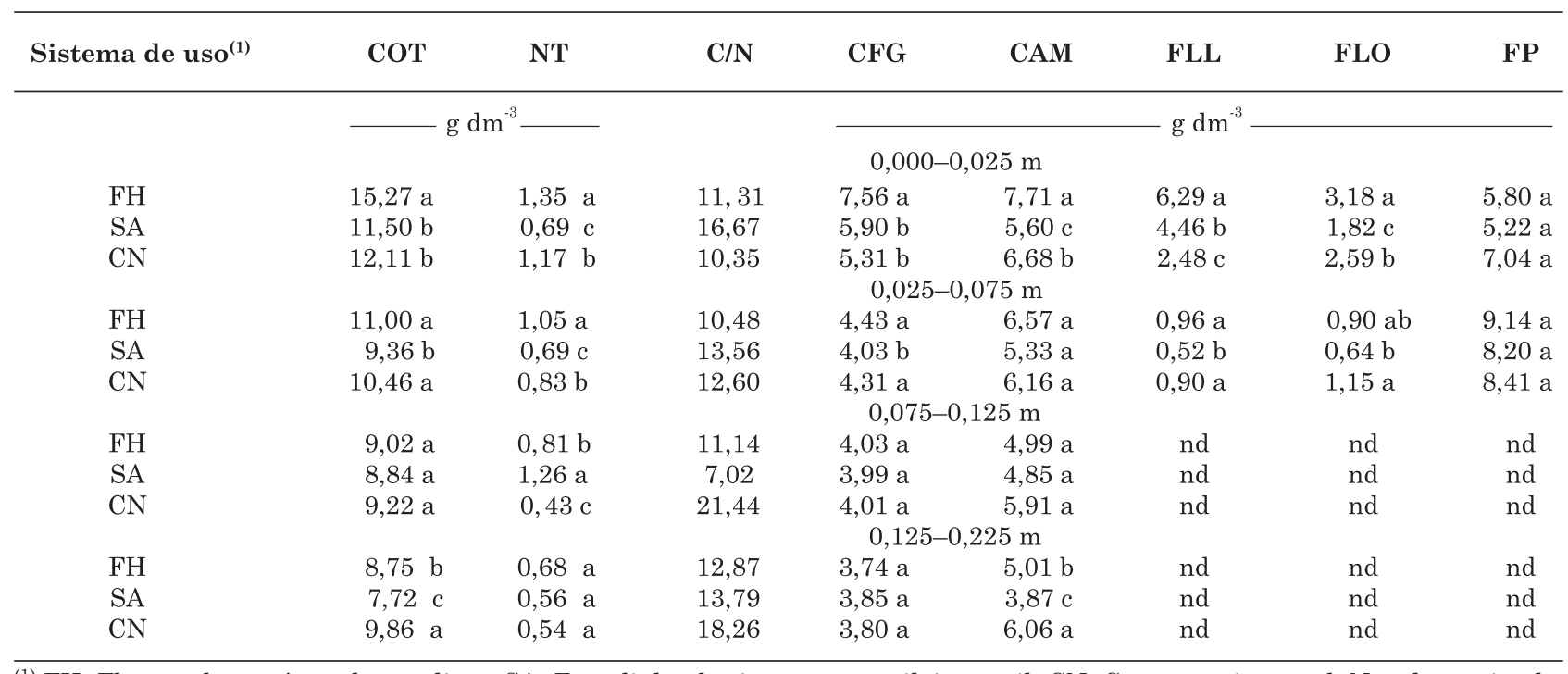

(1) FH: Floresta homogênea de eucalipto; SA: Entrelinha do sistema agrossilvipastoril; CN: Campo nativo; e nd: Não determinado. Médias seguidas pela mesma letra minúscula na coluna em cada camada não diferem entre si, pelo teste t, que considera diferença mínima significativa a $5 \%$.

não observaram diferenças nos teores de COT em mata nativa substituída por eucalipto. A mesma tendência foi verificada por Mendham et al. (2004) quando compararam solo sob eucalipto e pastagem. Esses autores observaram teor de CFG maior em solo sob eucalipto, comparativamente à pastagem, indicando alto potencial do primeiro em aportar resíduos culturais na superfície do solo, o que é atribuído à natureza física e química da serapilheira de eucalipto oriundo, principalmente, de materiais menos suscetíveis à degradação, devido à maior quantidade de lignina e tanino em relação às plantas forrageiras.

$\mathrm{Na}$ camada de 0,025-0,075 m, a FLL da FH e o $\mathrm{CN}$ foram superiores ao SA, resultante do cultivo ocorrido na entrelinha do SA nos primeiros dois anos após a instalação desse sistema. Lima et al. (2008) observaram, em Latossolo, maiores teores de C na FLL no solo sob cultivo de eucalipto até $0,10 \mathrm{~m}$, em comparação com o solo sob pastagem. Os teores de $\mathrm{C}$ na FLO na camada de 0,025-0,075 m decresceram na ordem $\mathrm{CN}>\mathrm{FH}>\mathrm{SA}$, indicando que sistemas naturais, de baixa intervenção antrópica, possuem maior capacidade para proteger fisicamente a MO no interior de agregados. Nesse caso, a FLO mostrou-se indicador sensível ao uso em solo de textura arenosa.

Não foram observadas diferenças nos teores de CAM nas camadas de 0,025 a 0,125 m (Quadro 3). Entretanto, na camada de 0,125 a $0,225 \mathrm{~m}$ os teores de $\mathrm{CAM}$ decresceram na ordem $\mathrm{CN}>\mathrm{FH}>\mathrm{SA}$. O maior teor de CAM observado no $\mathrm{CN}$ pode estar relacionado aos mecanismos de proteção química da MO, já que nesta camada verificou-se o maior teor de argila.
Os teores de C da FP não foram influenciados pelos sistemas de uso em todas as camadas (Quadro 3), possivelmente em decorrência do curto período de tempo de instalação dos sistemas (cinco anos). Essa fração é constituída por materiais orgânicos, fortemente ligados à fração mineral que se encontra em estádio avançado de humificação, sendo altamente estáveis devido à sua estabilidade química, pela interação com a fração mineral e a proteção física pela sua localização no interior de microagregados estáveis do solo, além de serem intrinsecamente recalcitrantes (Bayer, 1996). Como a MO da FP apresenta ciclagem lenta, é necessário um período maior para que sejam detectadas alterações devido aos sistemas de manejo (Bayer et al., 2004).

Observando as proporções de cada fração com relação ao COT do solo (Figura 2), pode-se inferir sobre a magnitude dos mecanismos de estabilidade da MO. Com exceção do campo nativo $(\mathrm{CN})$, na camada superficial a FLL representou, em média, na FH e no $\mathrm{SA}, 40 \%$ do teor de COT do solo, e a FP representou $41,5 \%$, indicando que o mecanismo de recalcitrância molecular da MO e a interação do material orgânico com os minerais do solo têm elevada importância diante da estabilidade decorrente da oclusão em agregados (FLO 18,5\%). Fato esse que pode estar relacionado, possivelmente, ao elevado teor de partículas de areia, influenciando, dessa forma, na formação de agregados estáveis e afetando a proteção física da MO. Embora essa distribuição seja coerente com a baixa capacidade de proteção química ou física que a matriz mineral oferece devido ao baixo teor de 
argila, ela impõe elevado risco à degradação, caso as adições de resíduos sejam suprimidas e, ou, o solo revolvido pelo preparo. Frações lábeis da MO são fundamentais para a ciclagem de $\mathrm{C}$ entre os compartimentos e para a ciclagem de nutrientes em curto prazo, além da contribuição para a formação e estabilização de agregados. Entretanto, essa fração é altamente sensível às alterações no uso e pode ser facilmente perdida por uso inadequado nos primeiros anos de cultivo (Mielniczuk, 2008).

Para a camada de 0,025-0,075 m, o C da FP esteve mais atuante em todos os sistemas de uso (Figura 2). Fato esse que pode ser explicado pela deposição de resíduos culturais em superfície, afetando a proporção de FLL em camadas mais profundas.

Os espectros de FTIR para as amostras das frações FLL e FLO mostraram-se semelhantes para os sistemas de uso (Figura 3). As seguintes bandas e respectivas atribuições foram identificadas: bandas de absorção em $3.266 \mathrm{~cm}^{-1}$, atribuídas a grupamentos $\mathrm{OH}$ ligados a estruturas aromáticas que compõem a MO e as substâncias húmicas presentes no solo (Silva et al., 2008), além de estiramento vibracional (v) de grupos AlO-H de argilas e oxi-hidróxidos (v FeO-H e u $\mathrm{SiO}-\mathrm{H}$ de argilas) (Nayak \& Singh, 2007). Nas regiões de 2.920 e $2.850 \mathrm{~cm}^{-1}$, encontram-se duas bandas

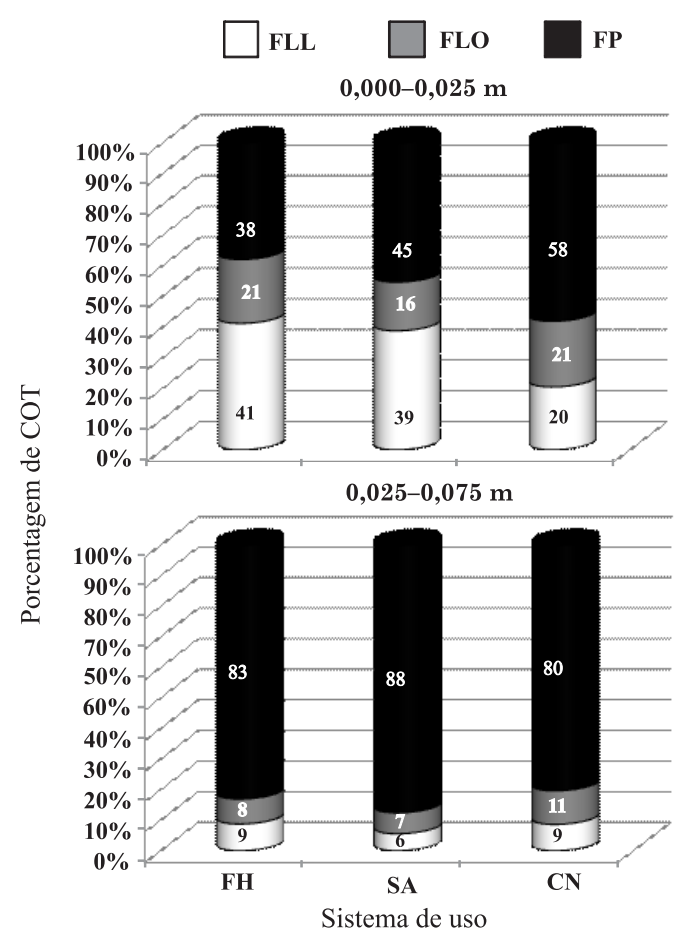

Figura 2. Proporção de carbono da fração leve livre (FLL), fração leve oclusa (FLO) e fração pesada (FP) de um Argissolo Vermelho submetido a diferentes sistemas de uso e camadas. FH: Floresta homogênea de eucalipto; SA: Sistema agrossilvipastoril na entrelinha; e CN: Campo nativo.

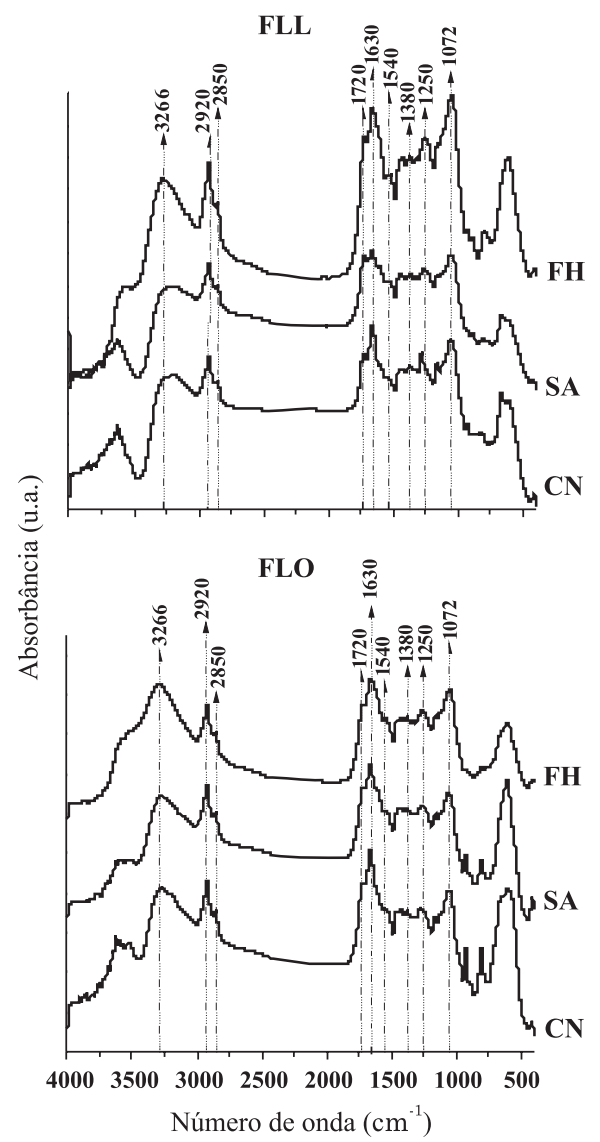

Figura 3. Espectros de Infravermelho com Transformada de Fourier (FTIR) das frações leve livre (FLL) e fração leve oclusa (FLO) da matéria orgânica de um Argissolo Vermelho submetido a diferentes sistemas de uso na camada de 0,000 0,025 m. FH: Floresta homogênea de eucalipto; SA: Sistema agrossilvipastoril na entrelinha; $\mathrm{e}$ CN: Campo nativo.

referentes ao estiramento dos grupos $\mathrm{C}$-H alifáticos de ésteres ou $\mathrm{C}=\mathrm{O}$ de cetonas (Figura 3 ). A banda em $1.720 \mathrm{~cm}^{-1}$ é atribuída ao estiramento $\mathrm{C}=\mathrm{O}$ do grupo carboxílico, e a banda em $1.630 \mathrm{~cm}^{-1}$ refere-se à vibração $\mathrm{C}=\mathrm{C}$ aromática, com contribuição de $\mathrm{C}=\mathrm{O}$ de amida, quinona e, ou, $\mathrm{C}=\mathrm{O}$ de grupos $\mathrm{COO}$. Em $1.540 \mathrm{~cm}^{-1}$, observou-se banda característica de estiramento C-C de anéis aromáticos, indicando a presença de lignina (Dobbss et al., 2009). Essa composição foi semelhante em todos os espectros, sendo típica de MO humificada (Silva et al., 2008). A banda pouco intensa observada na região entre $1.300 \mathrm{e}$ $1.400 \mathrm{~cm}^{-1}$ foi atribuída à ligação C-H alifático. Em $1.250 \mathrm{~cm}^{-1}$, verificaram-se banda característica de estiramento $\mathrm{C}-\mathrm{O}$ e a deformação $\mathrm{OH}$ de grupo carboxílico, e a banda em $1.072 \mathrm{~cm}^{-1}$ foi atribuída à ligação C-O de polissacarídeos (Dick et al., 2008). As bandas de absorção de polissacarídeos na FLO apresentaram menores intensidades em relação às bandas da FLL. 
Freixo et al. (2002) justificaram menor intensidade da banda $1.072 \mathrm{~cm}^{-1}$ na FLO, em decorrência do fato de essa fração encontra-se em estádio maior de humificação em relação à FLL (Figura 3). Por meio dos espectros de EPR, observaram-se linhas estreitas $(\sim 3500 \mathrm{G})$ tanto para a FLL quanto para a FLO, sendo essas semelhantes entre os sistemas de uso do solo (Figura 4). Essas linhas são atribuídas à presença de radical livre orgânico (RLO). O sinal do RLO de MO detectáveis por meio do EPR é atribuído a estruturas decorrentes de ligações em sistema $\pi$ orgânico (KögelKnaber et al., 1991). Quanto maior a concentração desses radicais, maior a humificação da MO que geralmente é relacionada à formação de compostos aromáticos. Segundo Martin-Neto et al. (1998), o caráter mais humificado está relacionado com a formação de compostos mais condensados que favorecem a estabilização desses radicais. A partir dos espectros de EPR em $50 \mathrm{G}$, foram calculados os valores de densidade de spins (spin $\mathrm{g}^{-1}$ ) e o fator $\mathrm{g}$ para a FLL e FLO (Quadro 4). Os valores obtidos para o fator $\mathrm{g}$ foram semelhantes entre os sistemas de uso do solo e entre as frações da MO $(2,003)$. Segundo Pérez et al. (2004), os fatores g, em torno de 2,003, indicam que os RLO localizam-se próximos a átomos de oxigênio, possivelmente conjugados com vários anéis aromáticos na MO. Em média, entre os sistemas de uso do solo a densidade de spins da FLO foi um pouco mais alta em relação à FLL, indicando maior transformação de sua estrutura em decorrência do processo de humificação. Segundo Olendzki (2006), maiores valores de spin $\mathrm{g}^{-1}$ indicam que os anéis do tipo quinona estão sendo reduzidos a polifenóis, passando à RLO. Esse tipo de mecanismo favorece a maior humificação da MO. A menor concentração de RLO para a FLL e, portanto, o menor grau de humificação indicam menor recalcitrância da MO em nível molecular, o que é favorável à disponibilidade de $\mathrm{C}$ e energia aos microrganismos decompositores nas frações mais lábeis (Bayer et al., 2003). A menor concentração de RLO é consistente com o grau de humificação menos avançado da FLL, sendo esta
Quadro 4. Radical livre orgânico (RLO) e fator g da fração leve livre (FLL) e fração leve oclusa (FLO) da matéria orgânica de um Argissolo Vermelho sob sistemas de uso e camada de 0,000 a $0,025 \mathrm{~m}$

\begin{tabular}{ccc}
\hline Sistema $^{(1)}$ & RLO & Fator $\mathbf{~}$ \\
\hline & spins/g $\left(10^{16}\right)$ & \\
\cline { 2 - 3 } FH & 2,14 & \\
SA & 3,31 & 2,0034 \\
CN & 2,30 & 2,0033 \\
& & 2,0033 \\
FH & 5,35 & \\
SA & 3,68 & 2,0033 \\
CN & 4,72 & 2,0031 \\
& & 2,0032 \\
\hline
\end{tabular}

(1) FH: Floresta homogênea de eucalipto; SA: Sistema agrossilvipastoril na entrelinha; e CN: Campo nativo.

composta basicamente por resíduos culturais em estádio inicial de decomposição (Cambardella \& Elliot, 1992).

\section{CONCLUSÕES}

1. A agregação na camada de 0,025 a $0,075 \mathrm{~m}$ indicou maior degradação estrutural do solo na entrelinha do sistema agrossilvipastoril.

2. O cultivo homogêneo de eucalipto promoveu maiores teores de $\mathrm{C}$ orgânico e de $\mathrm{N}$ total, de $\mathrm{C}$ da fração grosseira e das frações leve livre e leve oclusa nas camadas de 0,000 a $0,025 \mathrm{~m}$ e de 0,025 a $0,075 \mathrm{~m}$, em comparação com a entrelinha do sistema agrossilvipastoril e campo nativo.

3. Na camada de 0,000 a $0,025 \mathrm{~m}$, os mecanismos de proteção da matéria orgânica por recalcitrância molecular e estabilização química estão sobrepondo a estabilidade decorrente da oclusão em agregados. $\mathrm{Na}$

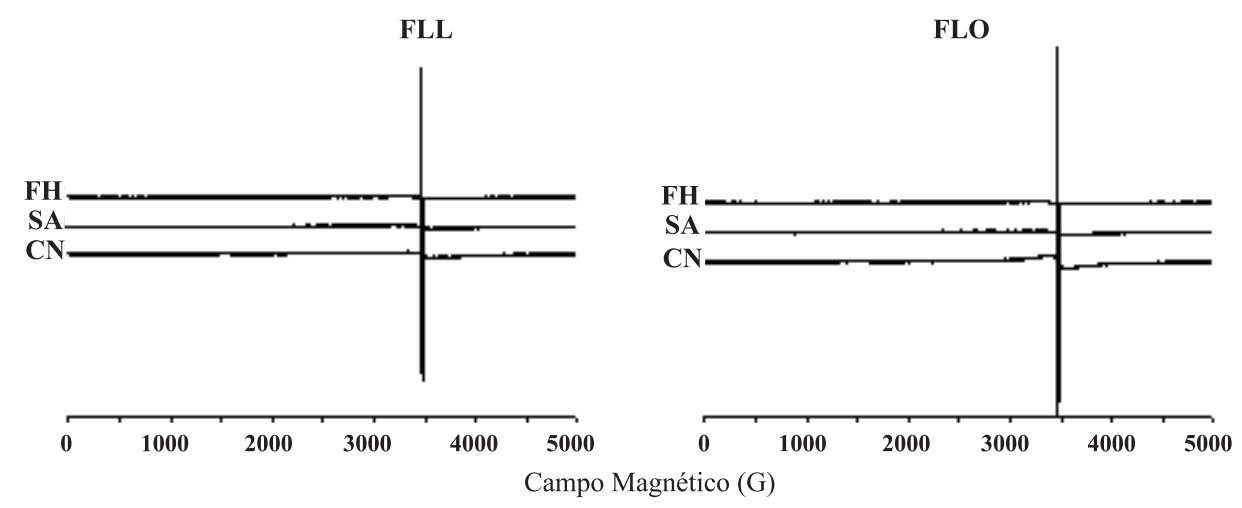

Figura 4. Espectros de Ressonância Paramagnética Eletrônica (EPR) da fração leve livre (FLL) e fração leve oclusa (FLO) da matéria orgânica de um Argissolo Vermelho submetido a sistemas de uso na camada de 0,000 a $0,025 \mathrm{~m}$. FH: Floresta homogênea de eucalipto; AS: Sistema agrossilvipastoril na entrelinha; e CN: Campo nativo. 
camada de 0,025 a $0,075 \mathrm{~m}$, a estabilidade da matéria orgânica deve, principalmente, à fração pesada.

4. Por meio das análises de Infravermelho com Transformada de Fourier, em $1.072 \mathrm{~cm}^{-1}$ observaramse bandas, atribuídas à presença de polissacarídeos, sendo elas menos intensas na fração leve oclusa em decorrência de essa fração se encontrar em estádio maior de humificação em relação à fração leve livre.

5. A fração leve oclusa apresentou maiores valores de radicais livres orgânicos do tipo semiquinona, indicando maior transformação de sua estrutura em decorrência do processo de humificação.

\section{LITERATURA CITADA}

BAYER, C. Dinâmica da matéria orgânica em sistemas de manejo de solos. Porto Alegre, Universidade Federal do Rio Grande do Sul, 1996. 240p. (Tese de Doutorado)

BAYER, C.; MARTIN-NETO L. \& SAAB, S.C. Diminuição da humificação da matéria orgânica de um Cambissolo Húmico em plantio direto. R. Bras. Ci. Solo, 27:537-544, 2003.

BAYER, C.; MARTIN-NETO, L.; MIELNICZUK, J. \& PAVINATO, A. Armazenamento de carbono em frações lábeis da matéria orgânica de um Latossolo vermelho sob plantio direto. Pesq. Agropec. Bras., 39:677-683, 2004.

CAMBARDELLA, C.A. \& ELLIOTT, E.T. Particulate soil organic-matter changes across a grassland cultivation sequence. Soil Sci. Soc. Am. J., 56:777-783, 1992.

CERETTA, C.A.; BAYER, C.; DICK, D.P.; MARTIN-NETO, L. \& COLNAGO, L.A. Métodos espectroscópico. In: SANTOS, G.A.; SILVA, L.S.; CANELLAS, L.P. \& CAMARGO, F.A.O., eds. Fundamentos da matéria orgânica do solo: Ecossistemas tropicais e subtropicais. 2.ed. Porto Alegre, Metrópole, 2008. p.201-227.

CONCEIÇÃO, P.C.; BOENI, M.; DIECKOW, J.; BAYER, C. \& MIELNICZUK, J. Fracionamento densimétrico com politungstato de sódio no estudo da proteção física da matéria orgânica em solos. R. Bras. Ci. Solo, 32:541-549, 2008.

DICK, D.P.; GONÇALVES, C.N.; DALMOLIN, R.S.D.; KNICKER, H.; KLAMT, E.; KÖGEL-KNABER, I.; SIMÕES, M.L. \& MARTIN-NETO, L. Characteristics of soil organic matter of different Brazilian Ferralsols under native vegetation as a function of soil depth. Geoderma, 124:319-333, 2005.

DICK, D.P.; MARTINAZZO, R.; DALMOLIN, R.S.D.; JACQUES, A.V.A.; MIELNICZUK, J. \& ROSA, A.S. Impacto da queima nos atributos químicos e na composição química da matéria orgânica do solo e na vegetação. Pesq. Agropec. Bras., 43:633-640, 2008.

DOBBSS, L.B.; RUMJANECK, V.M.; BALDOTTO, M.A.; VELLOSO, A.C.X. \& CANELLAS, L.P. Caracterização química e espectroscópica de ácidos húmicos e fúlvicos isolados da camada superficial de Latossolos brasileiros. R. Bras. Ci. Solo, 33:51-63, 2009.
EMPRESA BRASILEIRA DE PESQUISA AGROPECUÁRIA EMBRAPA. Centro Nacional de Pesquisa de Solos. Manual de métodos de análises de solo. Rio de Janeiro, 1997. 80p.

EMPRESA BRASILEIRA DE PESQUISA AGROPECUÁRIA EMBRAPA. Centro Nacional de Pesquisa de Solos. Sistema brasileiro de classificação de solos. 2.ed. Rio de Janeiro, Embrapa Solos, 2006. 306p.

FREIXO, A.A.; CANELLAS,L.P. \& MACHADO, P.L.O.A. Propriedades espectrais da matéria orgânica leve-livre e leve intra-agregados de dois Latossolos sob plantio direto e preparo convencional. R. Bras. Ci.Solo, 26:445-453, 2002.

GOLCHIN, A.; OADES, J.M.; SKJEMSTAD, J.O. \& CLARKE, P. Study of free and occluded particulate organic matter in soils by solid state 13C CP/MAS NMR spectroscopy and scanning electron microscopy. Autr. J.Soil Res., 32:285309,1994

GUO, L.B. \& GIFFORD, R.M. Soil carbon stocks and use change: A meta analysis. Global Change Biol., 8:345-360, 2002 .

KÖGEL-KNABER, I.; ZECH, W. \& HATCHER, P.G. Chemical structural studies of florest soil humic aromatics: aromatic carbon fraction. Soil Sci. Soc. Am. J., 55:241-247, 1991.

LIMA, A.M.N.; SILVA, I.R.; NEVES, J.C.L.; NOVAIS, R.F.; BARROS, N.F.; MENDONÇA, E.S.; DEMOLINARI, M.S.M. \& LEITE, F.P. Frações da matéria orgânica do solo após três décadas de cultivo de eucalipto no Vale do Rio Doce, MG. R. Bras. Ci. Solo, 32:1053-1063, 2008.

MARTIN-NETO, L.; ANDRIULO, A.E.; TRAGHETTA, D.G. Effects of cultivation on ESR spectra of organic matter from soil size fractions of a Mollisol. Soil Sci., 157:365372,1994

MARTIN-NETO, L.; NASCIMENTO, O.R.; TALAMONI, J. \& POPPI, N.R. EPR of micronutrientes-humic substances complexes extracted from a brazilian soil. Soil Sci., 151:369-376, 1991

MARTIN-NETO, L.; ROSSEL, R. \& SPOSITO, G. Correlation of spectroscopic indicators of humification with mean annual rainfall along a temperate grassland climossequence. Geoderma, 81:305-311, 1998.

MENDHAM, D.S.; HEAGNEY, E.C.; CORBEELS, M.; O'CONNELL, A.M.; GROVE, T.S. \& McMURTRIE, R.E. Soil particulate organic matter effects on nitrogen availability after afforestation with Eucalyptus globulus. Soil Biol. Biochem., 36:1067-1074, 2004.

MIELNICZUK, J. Matéria orgânica e sustentabilidade de sistemas agrícolas. In: SANTOS, G.A.; SILVA, L.S.; CANELLAS, L.P. \& CAMARGO, F.A.O., eds. Fundamentos da matéria orgânica do solo: Ecossistemas tropicais e subtropicais. 2.ed. Porto Alegre, Metrópole, 2008. p.1-5.

NAYAK, P.S. \& SINGH, K. Instrumental characterization of clay by XRF, XRD and FTIR. Bull. Mater Sci., 30:235-238, 2007. 
NEVES, C.M.N.; SILVA, M.L.; CURI, N.; MACEDO, R.L.G. \& TOKURA, A.M. Estoque de carbono em sistemas agrossilvipastoril, pastagem e eucalipto sob cultivo convencional na região noroeste do estado de Minas Gerais. R. Ci. Agrotec. 28:1038-1046, 2004.

OLENDZKI, R. N. Caracterização das interações de substâncias húmicas com a matéria inorgânica em solos de cultivo de arroz irrigado: contribuição à aquisição de dados essenciais para a avaliação global do seqüestro de carbono no solo. Curitiba, Universidade Federal do Paraná, 2006. 134p. (Tese de Doutorado)

PÉREZ,M.G.; MARTIN-NETO, L.; SAAB, S.C.; NOVOTNY, E.H.; MILORI, D.M.B.P.; BAGNATO, V.S.; COLNAGO, L.A.; MELO, W.J. \& KNICKER, H. Characterization of humic acids from a Brazilian Oxisol under different tillage systems by EPR, 13C NMR, FTIR and fluorescence spectroscopy. Geoderma, 18:181-190, 2004.

PULROLNIK, K.; BARROS, N.F.; SILVA, I.R.; NOVAIS, R.F. \& BRANDANI, C.B. Estoques de carbono e $\mathrm{N}$ em frações lábeis e estáveis da matéria orgânica de solos sob eucalipto, pastagem e cerrado no Vale do Jequitinhonha - MG. R. Bras. Ci. Solo, 33:1125-1136, 2009.

RIBASKI, J.; DEDECEK, R.A.; MATTEI, V.L.; FLORES, C.A.; VARGAS, A.F.C. \& RIBASKI, S.A.G. Sistemas silvipastoris: Estratégias para o desenvolvimento rural sustentável para a metade sul do Estado do Rio Grande do Sul. Colombo, Embrapa, 2005. (Comunicado Técnico, 150),
RIBASKI, S.A.G.; HOEFLICH, V.A. \& RIBASKI, J. Sistemas silvipastoris com apoio ao desenvolvimento rural para a Região Sudoeste do Rio Grande do Sul. Pesq. Flor. Bras., 60:27-37, 2009.

ROZANE, D.E.; CENTURION, J.F.; ROMUALDO, L.M.; TANIGUCHI, C.A.K.; TRABUCO, M. \& ALVES, A.U. Estoque de carbono e estabilidade de agregados de um Latossolo Vermelho distrófico, sob diferentes manejos. Biosci. J., 26:24-32, 2010.

RUSSELL, J.D. \& FRASER, A.R. Infrared methods. In: WILSON, M.J. Clay mineralogy: Spectroscopy and chemical determinative methods. London, Chapman \& Hall, 1994. p.18-61.

SALTON, J.C.; MIELNICZUK, J.; BAYER, C.; BOENI, M.; CONCEIÇÃO, P.C.; FABRÍCIO, A.C.; MACEDO, M.C.M. \& BROCH, D.L. Agregação e estabilidade de agregados do solo em sistemas agropecuários em Mato Grosso do Sul. R. Bras. Ci. Solo, 32:11-21, 2008.

SILVA, L.B.; DICK, D.P. \& INDA JÚNOR, A.V. Solos subtropicais de altitude: atributos químicos, teor de matéria orgânica e resistência à oxidação química. Ci. Rural, 38:1167-1171, 2008.

STEVENSON, J.F. Humus chemisthy, genesis, composition, reactions. 2.ed. New York, Wiley-Interscience Publication, 1994. 495p. 